\title{
Control landscapes for two-level open quantum systems
}

\author{
Alexander Pechen ${ }^{1 *}$, Dmitrii Prokhorenko ${ }^{2}$, Rebing $\mathrm{Wu}^{1}$ \\ and Herschel Rabitz ${ }^{1 \dagger}$
}

November 1, 2018

${ }^{1}$ Department of Chemistry, Princeton University, Princeton, New Jersey 08544, USA

2 Institute of Spectroscopy, Troitsk, Moscow Region 142190, Russia

\begin{abstract}
A quantum control landscape is defined as the physical objective as a function of the control variables. In this paper the control landscapes for two-level open quantum systems, whose evolution is described by general completely positive trace preserving maps (i.e., Kraus maps), are investigated in details. The objective function, which is the expectation value of a target system operator, is defined on the Stiefel manifold representing the space of Kraus maps. Three practically important properties of the objective function are found: (a) the absence of local maxima or minima (i.e., false traps); (b) the existence of multi-dimensional sub-manifolds of optimal solutions corresponding to the global maximum and minimum; and (c) the connectivity of each level set. All of the critical values and their associated critical sub-manifolds are explicitly found for any initial system state. Away from the absolute extrema there are no local maxima or minima, and only saddles may exist, whose number and the explicit structure of the corresponding critical sub-manifolds are determined by the initial system state. There are no saddles for pure initial states, one saddle for a completely mixed initial state, and two saddles for partially mixed initial states. In general, the landscape analysis of critical points and optimal manifolds is relevant to explain the relative ease of obtaining good optimal control outcomes in the laboratory, even in the presence of the environment.
\end{abstract}

\section{Introduction}

A common goal in quantum control is to maximize the expectation value of a given target operator by applying a suitable external action to the system. Such an external action often can be realized by a tailored coherent control field steering the system from the initial state to a target state, which maximizes the expectation value of the target operator [1, 2, 3, 4, 5, 6, 7, 8, 9]. Tailored coherent fields allow for controlling Hamiltonian aspects (i.e., unitary dynamics) of the system evolution. Another form of action on the system could

\footnotetext{
*E-mail: apechen@princeton.edu

${ }^{\dagger}$ E-mail: hrabitz@princeton.edu
} 
be realized by tailoring the environment (e.g., incoherent radiation, or a gas of electrons, atoms, or molecules) to induce control through non-unitary system dynamics [10]. In this approach the control is the suitably optimized, generally non-equilibrium and time dependent distribution function of the environment; the optimization of the environment would itself be attained by application of a proper external action. Combining such incoherent control by the environment (ICE) with a tailored coherent control field provides a general tool for manipulating both the Hamiltonian and dissipative aspects of the system dynamics. A similar approach to incoherent control was also suggested in [11] where, in difference with [10], finite-level ancilla systems are used as the control environment. The initial state of the field and the interaction Hamiltonian as the parameters for controlling non-unitary dynamics was also suggested in [12]. Non-unitary controlled quantum dynamics can also be realized by using as an external action suitably optimized quantum measurements which drive the system towards the desired control goal [13, 14, 15, 16, 17]. General mathematical definitions for the controlled Markov dynamics of quantum-mechanical systems are formulated in [18].

In this paper we consider the most general physically allowed transformations of states of quantum open systems, which are represented by completely positive trace preserving maps (i.e., Kraus maps) [19, 20, 21, 22]. A typical control problem in this framework is to find, for a given initial state of the system, a Kraus map which transforms the initial state into the state maximizing the expected value $\langle\Theta\rangle$ of a target operator $\Theta$ of the system. Practical means to find such optimal Kraus maps in the laboratory could employ various procedures such as adaptive learning algorithms [3, 23], which are capable of finding an optimal solution without detailed knowledge of the dynamics of the system. Kraus maps can be represented by matrices satisfying an orthogonality constraint (see Sec. II), which can be naturally parameterized by points in a Stiefel manifold [24], and then various algorithms may be applied to perform optimization over the Stiefel manifold (e.g., steepest descent, Newton methods, etc. adapted for optimization over Stiefel manifolds) [25, 26].

The quantum control landscape is defined as the objective expectation value $\langle\Theta\rangle$ as a function of the control variables. The efficiency of various search algorithms (i.e., employed either directly in the laboratory or in numerical simulations) for finding the minimum or maximum of a specific objective function can depend on the existence and nature of the landscape critical points. For example, the presence of many local minima or maxima (i.e., false traps) could result in either permanent trapping of the search or possibly dwelling for a long time in some of them (i.e., assuming that the algorithm has the capability of extricating the search from a trap) thus lowering the search efficiency. In such cases stopping of an algorithm at some solution does not guarantee that this solution is a global optimum, as the algorithm can end the search at a local maximum of the objective function. A priori information about absence of local maxima could be very helpful in such cases to guarantee that the search will be stopped only at a global optimum solution. This situation makes important the investigation of the critical points of the control landscapes. Also, in the laboratory, evidence shows that it is relatively easy to find optimal solutions, even in the presence of an environment. Explanation of this fact similarly can be related with the structure of the control landscapes for open quantum systems.

The critical points of the landscapes for closed quantum systems controlled by unitary evolution were investigated in $[27,28,29,30,31]$, where it was found that there are no sub- 
optimal local maxima or minima and only saddles may exist in addition to the global maxima and minima. In particular, it was found that for a two-level system prepared initially in a pure state the landscape of the unitary control does not have critical points except for global minima and maxima.

The capabilities of unitary control to maximize or minimize the expectation value of the target operator in the case of mixed initial states are limited, since unitary transformations can only connect states (i.e., density matrices) with the same spectrum. In going beyond the latter limitations, the dynamics may be extended to encompass non-unitary evolution by directing the controls to include the set of Kraus maps (i.e., dual manipulation of the system and the environment). Quantum systems which admit arbitrary Kraus map dynamics are completely controllable, since for any pair of states there exists a Kraus map which transforms one into the another [32].

In this paper the analysis of the landscape critical points is performed for two-level quantum systems controlled by Kraus maps. It is found that the objective function does not have sub-optimal local maxima or minima and only saddles may exist. The number of different saddle values and the structure of the corresponding critical sub-manifolds depend on the system initial state. For pure initial states the landscape has no saddles; for a completely mixed initial state the landscape has one saddle value; for other (i.e., partially mixed) initial states the landscape has two saddle values. For each case we explicitly find all critical sub-manifolds and critical values of the objective as functions of the Stokes vector of the initial density matrix. An investigation of the landscapes for multi-level open quantum systems with a different method may also be performed [36]. The absence of local minima or maxima holds also in the general case although an explicit description of the critical manifolds is difficult to provide for multi-level systems. The absence of false traps practically implies the relative ease of obtaining good optimal solutions using various search algorithms in the laboratory, even in the presence of an environment.

It should be noted that the property of there being no false traps relies on the assumption of the full controllability of the system, i.e., assuming that an arbitrary Kraus map can be realized. Restrictions on the set of available Kraus maps can result in the appearance of false traps thus creating difficulties in the search for optimal solutions. Thus, it is important to consider possible methods for engineering arbitrary Kraus type evolution of a controlled system. One method is to put the system in contact with an ancilla and implement, on the coupled system, specific unitary evolution whose form is determined by the structure of the desired Kraus map [37] (see also Sec. II). Lloyd and Viola proposed another method of engineering arbitrary Kraus maps, based on the combination of coherent control and measurements [38]. They show that the ability to perform a simple single measurement on the system together with the ability to apply coherent control to feedback the measurement results allows for enacting arbitrary Kraus map evolution at a finite time.

A level set of the objective function is defined as the set of controls which produce the same outcome value for $\langle\Theta\rangle$. We investigate connectivity of the level sets of the objective functions for open quantum systems and show that each level set is connected, including the one which corresponds to the global maximum/minimum of the objective function. Connectivity of a level set implies that any two solutions from the same level set can be continuously mapped one into another via a pathway entirely passing through this level set. The proof of 


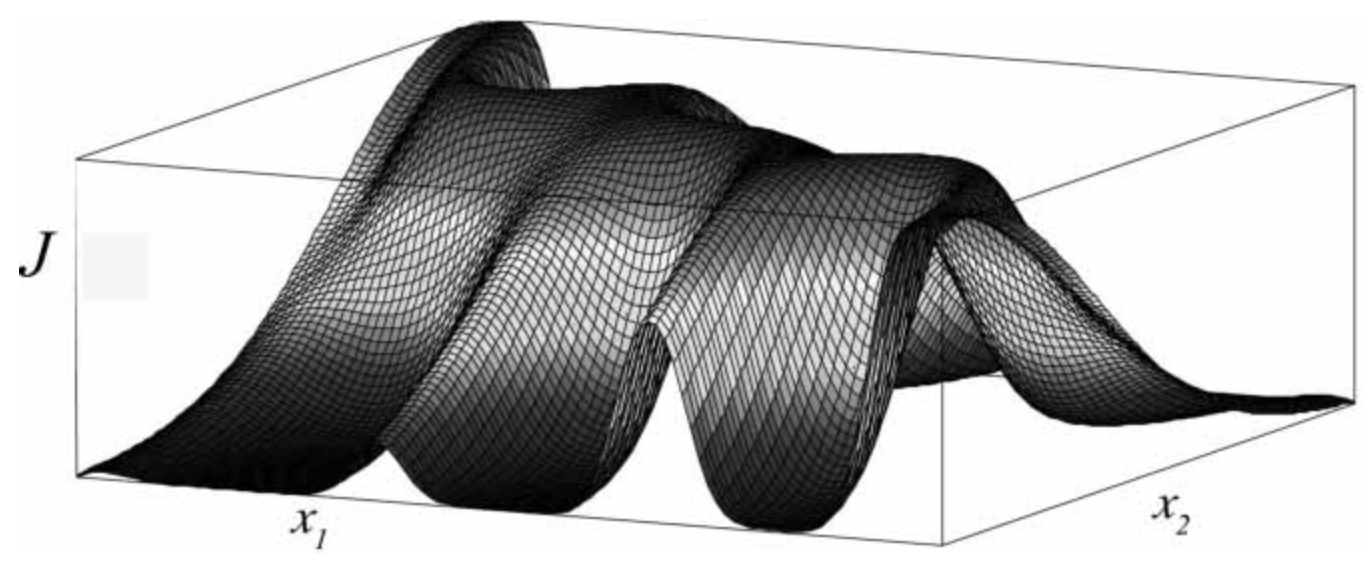

Figure 1: This figure schematically illustrates the landscape $J$ as a function of two controls $x_{1}$ and $x_{2}$. The figure shows the two main properties of quantum-mechanical control landscapes for open quantum systems: (a) absence of false traps and (b) connectivity of the sub-manifold of global maximum solutions (a one dimensional curve at the top of the landscape in this example).

the connectivity of the level sets is based on a generalization of Morse theory. Experimental observations of level sets for quantum control landscapes can be practically performed, as it was recently demonstrated for control of nonresonant two-photon excitations [39].

In summary, the main properties of control landscapes for open quantum systems are: (a) the absence of false traps; (b) the existence of multi-dimensional sub-manifolds of global optimum solutions, and (c) the connectivity of each level set. The proof of the properties (a)(c) is provided in the next sections for the two-level case. Figure 1 illustrates the properties (a), (b), and connectivity of the manifold of global maximum solutions; the figure does not serve to illustrate other properties such as connectivity of each level set. It is evident that the function drawn on figure 1 does not have local minima or maxima and the set of solutions for the global maximum is a connected sub-manifold (a curve in this case). A simple illustration is chosen for the figure since an exact objective function for an $N$-level quantum system depends on $D=2 N^{4}-2 N^{2}$ real variables (such that $D=24$ for $N=2$ ) and therefore can not be drawn.

The present analysis is performed in the kinematic picture which uses Kraus maps to represent evolution of quantum open systems. An important future task is to investigate the structure of the control landscape in the dynamical picture, which can be based on the use of various dynamical master equations to describe the dynamics of quantum open systems [22, 40, 41, 42, 43, 44]. Such analysis may reveal landscape properties for quantum open systems under (possibly, restricted) control through manipulation by a specific type of the environment (e.g., incoherent radiation).

In addition to optimizing expected value of a target operator, a large class of quantum control problems includes generation of a predefined unitary (e.g., phase or Hadamard) [21] or a non-unitary [33] quantum gate (i.e., a quantum operation). This class of control problems is important for quantum computation and in this regard a numerical analysis of the problem of optimal controlled generation of unitary quantum gates for two-level quantum systems interacting with an environment is available [34, 35]. 
Although the assumption of complete positivity of the dynamics of open quantum systems used in the present analysis is a generally accepted requirement, some works consider dynamics of a more general form [45, 46]. Such more general evolutions may result in different controllability and landscape properties. For example, for a two-level open quantum system positive and completely positive dynamics may have different accessibility properties [47]. In this regard it would be interesting to investigate if such different types of the dynamics have distinct essential landscape properties.

In Sec. 2 the optimal control problem for a general $N$-level open quantum system is formulated. Section 3 reduces the consideration to the case of a two-level system. In Sec. 4 a complete description is given of all critical points of the control landscape. The connectivity of the level sets is investigated in Sec. 5 .

\section{Formulation for an $N$-level system}

Let $\mathcal{M}_{N}$ be the linear space of $N \times N$ complex matrices. The density matrix $\rho$ of an $N$-level quantum system is a positive component in $\mathcal{M}_{N}, \rho \geq 0$, with unit trace, $\operatorname{Tr} \rho=1$ (Hermicity of $\rho$ follows from its positivity). Physically allowed evolution transformations of density matrices are given by completely positive trace preserving maps (i.e., Kraus maps) in $\mathcal{M}_{N}$. A linear Kraus map $\Phi: \mathcal{M}_{N} \rightarrow \mathcal{M}_{N}$ satisfies the following conditions [19]:

- Complete positivity. Let $\mathbb{I}_{n}$ be the identity matrix in $\mathcal{M}_{n}$. Complete positivity means that for any integer $n \in \mathbb{N}$ the map $\Phi \otimes \mathbb{I}_{n}$ acting in the space $\mathcal{M}_{N} \otimes \mathcal{M}_{n}$ is positive.

- Trace preserving: $\forall \rho \in \mathcal{M}_{N}, \operatorname{Tr} \Phi(\rho)=\operatorname{Tr} \rho$.

Any Kraus map $\Phi$ can be decomposed (non-uniquely) in the Kraus form [48, 9]:

$$
\Phi(\rho)=\sum_{l=1}^{M} K_{l} \rho K_{l}^{\dagger},
$$

where the Kraus operators $K_{l}$ satisfy the relation $\sum_{l=1}^{M} K_{l}^{\dagger} K_{l}=\mathbb{I}_{N}$. For an $N$-level quantum system it is sufficient to consider at most $M=N^{2}$ Kraus operators [48].

Let $\mathcal{H}_{1}=\mathbb{C}^{N}$ be the Hilbert space of the system under control. An arbitrary Kraus map of the form (1) can be realized by coupling the system to an ancilla system characterized by the Hilbert space $\mathcal{H}_{2}=\mathbb{C}^{M}$, and generating a unitary evolution operator $U$ acting in the Hilbert space of the total system $\mathcal{H}=\mathcal{H}_{1} \otimes \mathcal{H}_{2}$ as follows [37]. Choose in $\mathcal{H}_{2}$ a unit vector $|0\rangle$ and an orthonormal basis $\left|e_{i}\right\rangle, i=1, \ldots, M$. For any $|\psi\rangle \in \mathcal{H}_{1}$ let $U(|\psi\rangle \otimes|0\rangle)=$ $\sum_{i=1}^{M} K_{i}|\psi\rangle \otimes\left|e_{i}\right\rangle$. Such an operator can be extended to a unitary operator in $\mathcal{H}$ and for any $\rho$ one has $\Phi(\rho)=\operatorname{Tr}_{\mathcal{H}_{2}}\left\{U(\rho \otimes|0\rangle\langle 0|) U^{\dagger}\right\}$. Therefore the ability to dynamically create, for example via coherent control, an arbitrary unitary evolution of the system and ancilla allows for generating arbitrary Kraus maps of the controlled system.

Let $\rho_{0}$ be the initial system density matrix. A typical optimization goal in quantum control is to maximize the expectation value $J=\langle\Theta\rangle$ of a target Hermitian operator $\Theta$ over an admissible set of dynamical transformations of the system density matrices. For coherent unitary control this expectation value becomes

$$
J[U]=\operatorname{Tr}\left[U \rho_{0} U^{\dagger} \Theta\right]
$$


where $U=U\left(t, t_{0}\right)$ is a unitary matrix, $U U^{\dagger}=U^{\dagger} U=\mathbb{I}_{N}$, which describes the evolution of the system during the control period from the initial time $t_{0}$ until some final time $t$ and implicitly incorporates the action of the coherent control field on the system.

In the present paper we consider general non-unitary controlled dynamics such that the controls are Kraus maps, for which the parametrization by Kraus operators is used. The corresponding objective function specifying the control landscape has the form

$$
J\left[K_{1}, \ldots, K_{M}\right]=\operatorname{Tr}\left[\sum_{l=1}^{M} K_{l} \rho_{0} K_{l}^{\dagger} \Theta\right]
$$

where the Kraus operators $\left\{K_{l}\right\}=\left\{K_{l}\left(t, t_{0}\right)\right\}$ describe evolution of the open quantum system from an initial time $t_{0}$ until some final time $t$. The control goal is to maximize the objective function over the set of all Kraus operators $K_{1}, \ldots, K_{M}$ satisfying $\sum_{l=1}^{M} K_{l}^{\dagger} K_{l}=\mathbb{I}_{N}$, thereby forming a constrained optimization problem.

Definition 1 Let $\mathbb{F}$ be a field of real or complex numbers, i.e., $\mathbb{F}=\mathbb{R}$ or $\mathbb{F}=\mathbb{C}$. A Stiefel manifold over $\mathbb{F}$, denoted $V_{k}\left(\mathbb{F}^{n}\right)$, is the set of all orthonormal $k$-frames in $\mathbb{F}^{n}$ (i.e., the set of ordered $k$-tuples of orthonormal vectors in $\left.\mathbb{F}^{n}\right)$. The case $\mathbb{F}=\mathbb{R}$ (respectively, $\mathbb{F}=\mathbb{C}$ ) corresponds to a real (complex) Stiefel manifold.

Let $K$ be the $N \times(N M)$ matrix defined as $K=\left(K_{1}^{\mathrm{T}} \ldots K_{M}^{\mathrm{T}}\right)$, where $K_{l}^{\mathrm{T}}$ is the transpose of matrix $K_{l}$ and $M$ is the number of Kraus operators. Consider $N$ vectors $X_{1}, \ldots, X_{N} \in \mathbb{C}^{N M}$ with components $\left(X_{i}\right)_{j}=K_{i j}$, i.e., vector $X_{i}$ is the $i$-th row of the matrix $K$. The constraint $\sum_{l=1}^{M} K_{l}^{\dagger} K_{l}=\mathbb{I}_{N}$ in terms of the vectors $X_{1}, \ldots, X_{N}$ takes the form $\left\langle X_{i}, X_{j}\right\rangle=\delta_{i j}$, where $\delta_{i j}$ is the Kronecker delta symbol. This constraint defines the complex Stiefel manifold $V_{N}\left(\mathbb{C}^{N M}\right)$. Therefore optimization of the objective function $J\left[K_{1}, \ldots, K_{M}\right]$ defined by Eq. (2) can be formulated as optimization over the complex Stiefel manifold $V_{N}\left(\mathbb{C}^{N M}\right)$.

\section{Two-level system}

In the following we consider the case of a two-level system in detail. Any density matrix of a two-level system can be represented as

$$
\rho=\frac{1}{2}[1+\langle\mathbf{w}, \sigma\rangle]
$$

where $\sigma=\left(\sigma_{1}, \sigma_{2}, \sigma_{3}\right) \equiv\left(\sigma_{x}, \sigma_{y}, \sigma_{z}\right)$ is the vector of Pauli matrices and $\mathbf{w} \in \mathbb{R}^{3}$ is the Stokes vector, $\|\mathbf{w}\| \leq 1$. Thus, the set of density matrices can be identified with the unit ball in $\mathbb{R}^{3}$, which is known as the Bloch sphere.

Any Kraus map $\Phi$ on $\mathcal{M}_{2}$ can be represented using at most four Kraus operators

$$
K_{l}=\left(\begin{array}{ll}
x_{l 1} & x_{l 3} \\
x_{l 2} & x_{l 4}
\end{array}\right), \quad l=1,2,3,4
$$

as $\Phi(\rho)=\sum_{l=1}^{4} K_{l} \rho K_{l}^{\dagger}$, where the Kraus operators satisfy the constraint

$$
\sum_{l=1}^{4} K_{l}^{\dagger} K_{l}=\mathbb{I}_{2}
$$


Let $\rho_{0}$ be the initial system density matrix with Stokes vector $\mathbf{w}=(\alpha, \beta, \gamma)$, where $\|\mathbf{w}\|^{2}=\alpha^{2}+\beta^{2}+\gamma^{2} \leq 1$, and let $\Theta$ be a Hermitian target operator. The objective functional for optimizing the expectation value of $\Theta$ has the form $J\left[K_{1}, K_{2}, K_{3}, K_{4} ; \rho_{0}, \Theta\right]=$ $\sum_{l=1}^{4} \operatorname{Tr}\left[K_{l} \rho_{0} K_{l}^{\dagger} \Theta\right]$. The control goal is to find all quadruples of Kraus operators $\left(K_{1}, K_{2}, K_{3}, K_{4}\right)$ which maximize (or minimize, depending on the control goal) the objective functional $J$. The goal of the landscape analysis is to characterize all critical points of $J\left[K_{1}, K_{2}, K_{3}, K_{4}\right]$, including local extrema, if they exist.

The analysis for an arbitrary $2 \times 2$ Hermitian matrix $\Theta$ can be reduced to the case

$$
\Theta_{0}=\left(\begin{array}{ll}
1 & 0 \\
0 & 0
\end{array}\right)
$$

which we will consider in the sequel. This point follows, as an arbitrary Hermitian operator $\Theta \in \mathcal{M}_{2}$ has two eigenvalues $\lambda_{1}$ and $\lambda_{2}$ and can be represented in the basis of its eigenvectors as

$$
\Theta=\left(\begin{array}{cc}
\lambda_{1} & 0 \\
0 & \lambda_{2}
\end{array}\right)
$$

where $\lambda_{1} \geq \lambda_{2}$. One has $\Theta=\left(\lambda_{1}-\lambda_{2}\right) \Theta_{0}+\lambda_{2} \mathbb{I}_{2}$ and

$$
\begin{aligned}
J\left[K_{1}, K_{2}, K_{3}, K_{4} ; \rho_{0}, \Theta\right] & =\sum_{l=1}^{4} \operatorname{Tr}\left[K_{l} \rho_{0} K_{l}^{\dagger} \Theta\right] \\
& =\left(\lambda_{1}-\lambda_{2}\right) \sum_{l=1}^{4} \operatorname{Tr}\left[K_{l} \rho_{0} K_{l}^{\dagger} \Theta_{0}\right]+\lambda_{2} \sum_{l=1}^{4} \operatorname{Tr}\left[K_{l} \rho_{0} K_{l}^{\dagger}\right] \\
& =\left(\lambda_{1}-\lambda_{2}\right) J\left[K_{1}, K_{2}, K_{3}, K_{4} ; \rho_{0}, \Theta_{0}\right]+\lambda_{2}
\end{aligned}
$$

Therefore, the objective function for a general observable operator $\Theta$ depends linearly on the objective function defined for $\Theta_{0}$. We denote $J\left[K_{1}, K_{2}, K_{3}, K_{4} ; \mathbf{w}\right]:=J\left[K_{1}, K_{2}, K_{3}, K_{4} ; \rho_{0}, \Theta_{0}\right]$. In the trivial case $\Theta=\mathbb{I}_{2}$ the landscape is completely flat and no further analysis is needed.

\section{The critical points of the objective function land- scape}

The Kraus operators for a two-level system can be parameterized by a pair of vectors $X, Y \in$ $\mathbb{C}^{8}=\mathbb{C}^{4} \oplus \mathbb{C}^{4}$ of the form $X=u_{1} \oplus v_{1}$ and $Y=u_{2} \oplus v_{2}$, where $u_{1}=\left(x_{11}, x_{21}, x_{31}, x_{41}\right)$, $v_{1}=\left(x_{12}, x_{22}, x_{32}, x_{42}\right), u_{2}=\left(x_{13}, x_{23}, x_{33}, x_{43}\right)$, and $v_{2}=\left(x_{14}, x_{24}, x_{34}, x_{44}\right)$. The objective function in terms of these vectors has the form

$$
J\left[u_{1}, u_{2}, v_{1}, v_{2} ; \mathbf{w}\right]=\frac{1}{2}\left[(1+\gamma)\left\|u_{1}\right\|^{2}+(1-\gamma)\left\|u_{2}\right\|^{2}+2 \operatorname{Re}\left[z_{0}\left\langle u_{1}, u_{2}\right\rangle\right]\right]
$$

where $z_{0}=\alpha-\mathrm{i} \beta,\langle\cdot, \cdot\rangle$ and $\|\cdot\|$ denote the standard inner product and the norm in $\mathbb{C}^{N}$ (here the numbers $\alpha, \beta, \gamma$ are the components of the Stokes vector $\mathbf{w}=(\alpha, \beta, \gamma)$ of the initial density matrix $\rho_{0}$, see Sec. 3). The constraint (3) in terms of the vectors $X$ and $Y$ has the 
form $\|X\|=\|Y\|=1,\langle X, Y\rangle=0$ and determines the Stiefel manifold $\mathcal{M}=V_{2}\left(\mathbb{C}^{8}\right)$. The matrix constraint (3) in terms of the vectors $u_{i}$ and $v_{i}$ has the form

$$
\begin{aligned}
& \Phi_{1}\left(u_{1}, u_{2}, v_{1}, v_{2}\right):=\left\|u_{1}\right\|^{2}+\left\|v_{1}\right\|^{2}-1=0 \\
& \Phi_{2}\left(u_{1}, u_{2}, v_{1}, v_{2}\right):=\left\|u_{2}\right\|^{2}+\left\|v_{2}\right\|^{2}-1=0 \\
& \Phi_{3}\left(u_{1}, u_{2}, v_{1}, v_{2}\right):=\left\langle u_{1}, u_{2}\right\rangle+\left\langle v_{1}, v_{2}\right\rangle=0
\end{aligned}
$$

If $z_{0} \neq 0$, then the objective function is diagonalized by introducing new coordinates $\left(\tilde{u}_{1}, \tilde{u}_{2}, \tilde{v}_{1}, \tilde{v}_{2}\right)$ in $\mathbb{C}^{16}$ according to the formulas

$$
\begin{array}{ll}
u_{1}=\mu \tilde{u}_{1}-\nu \tilde{u}_{2}, & u_{2}=\frac{z_{0}^{*}}{\left|z_{0}\right|} \nu \tilde{u}_{1}+\frac{z_{0}^{*}}{\left|z_{0}\right|} \mu \tilde{u}_{2} \\
v_{1}=\mu \tilde{v}_{1}-\nu \tilde{v}_{2}, & v_{2}=\frac{z_{0}^{*}}{\left|z_{0}\right|} \nu \tilde{v}_{1}+\frac{z_{0}^{*}}{\left|z_{0}\right|} \mu \tilde{v}_{2}
\end{array}
$$

where $\mu=\left|z_{0}\right| / \sqrt{2\|\mathbf{w}\|(\|\mathbf{w}\|-\gamma)}$ and $\nu=\left|z_{0}\right| / \sqrt{2\|\mathbf{w}\|(\|\mathbf{w}\|+\gamma)}$. The objective function in these coordinates has the form

$$
J[x ; \mathbf{w}]=\lambda_{+}\left\|\tilde{u}_{1}\right\|^{2}+\lambda_{-}\left\|\tilde{u}_{2}\right\|^{2}
$$

where $x=\left(\tilde{u}_{1}, \tilde{u}_{2}, \tilde{v}_{1}, \tilde{v}_{2}\right) \in \mathcal{M}$ and $\lambda_{ \pm}=(1 \pm\|\mathbf{w}\|) / 2$. If $z_{0}=0$ and $\gamma \geq 0$ (resp., $\left.\gamma<0\right)$, then the objective function (4) has the form (10) with $\tilde{u}_{i}=u_{i}, \tilde{v}_{i}=v_{i}$ for $i=1,2$ (resp., $\tilde{u}_{1}=u_{2}, \tilde{u}_{2}=u_{1}, \tilde{v}_{1}=v_{2}, \tilde{v}_{2}=v_{1}$ ). The constraints (5)-(7) in the new coordinates have the same form $\Phi_{i}\left(\tilde{u}_{1}, \tilde{u}_{2}, \tilde{v}_{1}, \tilde{v}_{2}\right)=0$ for $i=1,2,3$.

Theorem 1 Let $\mathbf{w}=(\alpha, \beta, \gamma) \in \mathbb{R}^{3}$ be a real vector such that $\|\mathbf{w}\| \leq 1$ and let $\lambda_{ \pm}=$ $(1 \pm\|\mathbf{w}\|) / 2$. For any such $\mathbf{w}$, the global maximum and minimum values of the objective function $J\left[\tilde{u}_{1}, \tilde{u}_{2}, \tilde{v}_{1}, \tilde{v}_{2} ; \mathbf{w}\right]=\lambda_{+}\left\|\tilde{u}_{1}\right\|^{2}+\lambda_{-}\left\|\tilde{u}_{2}\right\|^{2}$ are

$$
\begin{aligned}
\min _{\left(\tilde{u}_{1}, \tilde{u}_{2}, \tilde{v}_{1}, \tilde{v}_{2}\right) \in \mathcal{M}} J\left[\tilde{u}_{1}, \tilde{u}_{2}, \tilde{v}_{1}, \tilde{v}_{2} ; \mathbf{w}\right] & =0 \\
\max _{\left(\tilde{u}_{1}, \tilde{u}_{2}, \tilde{v}_{1}, \tilde{v}_{2}\right) \in \mathcal{M}} J\left[\tilde{u}_{1}, \tilde{u}_{2}, \tilde{v}_{1}, \tilde{v}_{2} ; \mathbf{w}\right] & =1 .
\end{aligned}
$$

The critical sub-manifolds and other critical values of $J$ in $\mathcal{M}$ are the following:

Case 1. $\mathbf{w}=0$ (the completely mixed initial state). The global minimum sub-manifold is $\mathcal{M}_{\min }^{(0,0,0)}=\left\{x \in \mathcal{M} \mid \tilde{u}_{1}=\tilde{u}_{2}=0\right\}$. The global maximum sub-manifold is $\mathcal{M}_{\max }^{(0,0) 0)}=\{x \in$ $\left.\mathcal{M} \mid \tilde{v}_{1}=\tilde{v}_{2}=0\right\}$. The objective function has one saddle value $J=1 / 2$ with the corresponding critical sub-manifold $\mathcal{M}_{\text {saddle }}^{(0,0,0)}=\left\{x \in \mathcal{M} \mid \tilde{u}_{2}=z \tilde{u}_{1}, \tilde{v}_{1}=-z^{*} \tilde{v}_{2}, z \in \mathbb{C}\right\} \bigcup\left\{x \in \mathcal{M} \mid \tilde{u}_{1}=\right.$ $\left.\tilde{v}_{2}=0\right\}$. The Hessian of $J$ at any point at $\mathcal{M}_{\text {saddle }}^{(0,0,0)}$ has $\nu_{+}=6$ positive, $\nu_{-}=6$ negative, and $\nu_{0}=16$ zero eigenvalues.

Case 2. $0<\|\mathbf{w}\|<1$ (a partially mixed initial state). The global minimum sub-manifold is $\mathcal{M}_{\text {min }}^{\mathrm{w}}=\left\{x \in \mathcal{M} \mid \tilde{u}_{1}=\tilde{u}_{2}=0\right\}$. The global maximum sub-manifold is $\mathcal{M}_{\max }^{\mathrm{w}}=\{x \in$ $\left.\mathcal{M} \mid \tilde{v}_{1}=\tilde{v}_{2}=0\right\}$. The objective function has two saddle values:

$$
J_{ \pm}(\mathbf{w})=\frac{1 \pm\|\mathbf{w}\|}{2}=\lambda_{ \pm}
$$


The corresponding critical sub-manifolds are $\mathcal{M}_{-}^{\mathbf{w}}=\left\{x \in \mathcal{M} \mid \tilde{u}_{1}=\tilde{v}_{2}=0\right\}$ and $\mathcal{M}_{+}^{\mathbf{w}}=\{x \in$ $\left.\mathcal{M} \mid \tilde{u}_{2}=\tilde{v}_{1}=0\right\}$. The Hessian of $J$ at any point at $\mathcal{M}_{-}^{\mathbf{w}}$ (resp., $\mathcal{M}_{+}^{\mathbf{w}}$ ) has $\nu_{+}=8$ positive, $\nu_{-}=6$ negative (resp., $\nu_{+}=6$ positive, $\nu_{-}=8$ negative), and $\nu_{0}=14$ zero eigenvalues.

Case 3. $\|\mathbf{w}\|=1$ (a pure initial state). The global minimum sub-manifold is $\mathcal{M}_{\min }^{\mathbf{w}}=$ $\left\{x \in \mathcal{M} \mid \tilde{u}_{1}=0\right\}$. The global maximum sub-manifold is $\mathcal{M}_{\max }^{\mathbf{w}}=\left\{x \in \mathcal{M} \mid \tilde{v}_{1}=0\right\}$. The objective function has no saddles.

Proof. The objective function has the form $J=\rho_{11}$, where $\rho_{11}$ is the diagonal matrix element of the density matrix. Therefore $0 \leq J \leq 1$ and the value $J=0$ (resp., $J=1)$ corresponds to the global minimum (resp., maximum).

The constraints can be included in the objective function (10) by adding the term $\Phi[\tilde{u}, \tilde{v}, \eta]=\eta_{1} \Phi_{1}+\eta_{2} \Phi_{2}+2 \operatorname{Re}\left[\eta_{3}^{*} \Phi_{3}\right]$, where the two real and one complex Lagrange multipliers $\eta_{1}, \eta_{2}$, and $\eta_{3}$ correspond to the two real and one complex valued constraints $\Phi_{1}, \Phi_{2}$, and $\Phi_{3}$, respectively. Critical points of the function $J$ on the manifold $\mathcal{M}$ are given by the solutions of the following Euler-Lagrange equations for the functional $\widetilde{J}[\tilde{u}, \tilde{v}, \lambda]=$ $J[\tilde{u}, \tilde{v}]+\Phi[\tilde{u}, \tilde{v}, \eta]:$

$$
\begin{array}{ll}
0=\nabla_{\tilde{u}_{1}^{*}} \widetilde{J} \Rightarrow & 0=\left(\lambda_{+}+\eta_{1}\right) \tilde{u}_{1}+\eta_{3} \tilde{u}_{2} \\
0=\nabla_{\tilde{u}_{2}^{*}} \widetilde{J} \Rightarrow & 0=\eta_{3}^{*} \tilde{u}_{1}+\left(\lambda_{-}+\eta_{2}\right) \tilde{u}_{2} \\
0=\nabla_{\tilde{v}_{1}^{*}} \widetilde{J} \Rightarrow & 0=\eta_{1} \tilde{v}_{1}+\eta_{3} \tilde{v}_{2} \\
0=\nabla_{\tilde{v}_{2}^{*}} \widetilde{J} \Rightarrow & 0=\eta_{3}^{*} \tilde{v}_{1}+\eta_{2} \tilde{v}_{2}
\end{array}
$$

where $\tilde{u}_{1}, \tilde{u}_{2}, \tilde{v}_{1}, \tilde{v}_{2}$ satisfy the constraints (5)-(7). The proof of the theorem is based on the straightforward solution of the system (12)-(15). The case 2 will be considered first, followed by the cases 1 and 3 .

Case 2. $0<\|\mathbf{w}\|<1$. Consider in $\mathcal{M}$ the open subset $\mathcal{O}_{1}=\left\{x \in \mathcal{M} \mid \tilde{v}_{1} \neq 0, \tilde{v}_{2} \neq 0\right\}$. Let us prove that the set of all critical points of $J$ in $\mathcal{O}_{1}$ is the set of all points of $\mathcal{M}$ such that $\tilde{u}_{1}=\tilde{u}_{2}=0$.

Suppose that there are critical points in $\mathcal{O}_{1}$ such that $\tilde{u}_{1} \neq 0$ or $\tilde{u}_{2} \neq 0$. For such points the following identity holds

$$
\left|\eta_{3}\right|^{2}=\left(\lambda_{+}+\eta_{1}\right)\left(\lambda_{-}+\eta_{2}\right)
$$

In $\mathcal{O}_{1}, \tilde{v}_{1} \neq 0$ and therefore $\left|\eta_{3}\right|^{2}=\eta_{1} \eta_{2}$. This equality together with $(16)$ gives

$$
\eta_{2}=-\lambda_{-}\left(1+\frac{\eta_{1}}{\lambda_{+}}\right)
$$

Suppose that $\eta_{3} \neq 0$. Then, using $(12)$ and (14), the constraint $\Phi_{3}$ gives

$$
\left(\lambda_{+}+\eta_{1}\right)\left\|\tilde{u}_{1}\right\|^{2}+\eta_{1}\left\|\tilde{v}_{1}\right\|^{2}=0 .
$$

Constraint $\Phi_{1}$ gives $\left\|\tilde{v}_{1}\right\|^{2}=1-\left\|\tilde{u}_{1}\right\|^{2}$, and therefore $\eta_{1}=-\lambda_{+}\left\|\tilde{u}_{1}\right\|^{2}$. Similarly we find $\eta_{2}=-\lambda_{-}\left\|\tilde{u}_{2}\right\|^{2}$. Substituting these expressions for $\eta_{1}$ and $\eta_{2}$ into the (12) and (13) we find

$$
\left\{\begin{array} { l } 
{ \sqrt { \lambda _ { - } \lambda _ { + } } \| \tilde { u } _ { 1 } \| \| \tilde { u } _ { 2 } \| ^ { 2 } = \lambda _ { + } ( 1 - \| \tilde { u } _ { 1 } \| ^ { 2 } ) \| \tilde { u } _ { 1 } \| } \\
{ \sqrt { \lambda _ { - } \lambda _ { + } } \| \tilde { u } _ { 1 } \| ^ { 2 } \| \tilde { u } _ { 2 } \| = \lambda _ { - } ( 1 - \| \tilde { u } _ { 2 } \| ^ { 2 } ) \| \tilde { u } _ { 2 } \| }
\end{array} \Rightarrow \left\{\begin{array}{l}
\sqrt{\lambda_{+}}=\sqrt{\lambda_{+}}\left\|\tilde{u}_{1}\right\|^{2}+\sqrt{\lambda_{-}}\left\|\tilde{u}_{2}\right\|^{2} \\
\sqrt{\lambda_{-}}=\sqrt{\lambda_{+}}\left\|\tilde{u}_{1}\right\|^{2}+\sqrt{\lambda_{-}}\left\|\tilde{u}_{2}\right\|^{2}
\end{array}\right.\right.
$$


This system of equations implies $\lambda_{-}=\lambda_{+} \Leftrightarrow \mathbf{w}=0$ which is in contradiction with the assumption $\|\mathbf{w}\|>0$ for the present case. If $\eta_{3}=0$, then it follows from (14), (15) that $\eta_{1}=\eta_{2}=0$. In this case equations $(12)$ and $(13)$ have only the solution $\tilde{u}_{1}=\tilde{u}_{2}=0$.

Points in $\mathcal{O}_{1}$ with $\tilde{u}_{1}=\tilde{u}_{2}=0$ form the global minimum manifold $\mathcal{M}_{\text {min }}^{\mathrm{w}}=V_{2}\left(\mathbb{C}^{4}\right)$, which is a Stiefel manifold and hence is connected. In some small neighborhood of zero we can choose $\tilde{u}_{1}$ and $\tilde{u}_{2}$ as normal coordinates. So $\mathcal{M}_{\mathrm{min}}^{\mathbf{w}}$ is non degenerate. Similar treatment of the region $\mathcal{O}_{2}=\left\{x \in \mathcal{M} \mid \tilde{u}_{1} \neq 0, \tilde{u}_{2} \neq 0\right\}$ gives the global maximum manifold $\mathcal{M}_{\max }^{\mathrm{w}}=\left\{x \in \mathcal{M} \mid \tilde{v}_{1}=\tilde{v}_{2}=0\right\}$.

Now consider the region $\mathcal{O}_{3}=\left\{x \in \mathcal{M} \mid \tilde{u}_{2} \neq 0, \tilde{v}_{1} \neq 0\right\}$. In this region the objective function $J$ has the form

$$
J\left[\tilde{u}_{1}, \tilde{u}_{2}, \tilde{v}_{1}, \tilde{v}_{2}\right]=\lambda_{-}+\lambda_{+}\left\|\tilde{u}_{1}\right\|^{2}-\lambda_{-}\left\|\tilde{v}_{2}\right\|^{2} .
$$

Using the analysis for the region $\mathcal{O}_{1}$, we conclude that the objective function has no critical points such that $\tilde{v}_{2} \neq 0$ in $\mathcal{O}_{3}$. Therefore all critical points in $\mathcal{O}_{3}$ are in the sub-manifold $\mathcal{N}=\left\{x \in \mathcal{M} \mid \tilde{v}_{2}=0\right\} \subset \mathcal{M}$. The restriction of $J$ to $\mathcal{N}$ has the form

$$
\left.J\left[\tilde{u}_{1}, \tilde{u}_{2}, \tilde{v}_{1}, \tilde{v}_{2}\right]\right|_{\mathcal{N}}=\lambda_{-}+\lambda_{+}\left\|\tilde{u}_{1}\right\|^{2}
$$

Note that $\mathcal{N}$ is a subset of all sets of vectors $\left(\tilde{u}_{1}, \tilde{u}_{2}, \tilde{v}_{1}\right)$ satisfying the constraints

$$
\left\|\tilde{u}_{2}\right\|^{2}=1, \quad\left\|\tilde{u}_{1}\right\|^{2}+\left\|\tilde{v}_{1}\right\|^{2}=1, \quad\left\langle\tilde{u}_{1}, \tilde{u}_{2}\right\rangle=0 .
$$

It is clear from this representation of $\mathcal{N}$ that $\left.\nabla J\right|_{\mathcal{N}}=0$ if and only if $\tilde{u}_{1}=0$. This gives the critical sub-manifold $\mathcal{M}_{-}^{\mathbf{w}}=\left\{x \in \mathcal{M} \mid \tilde{u}_{1}=\tilde{v}_{2}=0\right\}$. The objective function has the value $\left.J\right|_{\mathcal{M}_{-}}=\lambda_{-}$on this manifold.

To show that this is a saddle manifold, and not a local maximum or minimum, we calculate the Morse indices of the objective function on $\mathcal{M}_{-}^{\mathbf{w}}$ and show that both positive and negative Morse indices are different from zero (the Morse indices are the numbers of positive, negative and zero eigenvalues of the Hessian of $J$ and positive and negative Morse indices determine the number of local coordinates along which the function increases or decreases, respectively). With regard to this goal, consider the manifold $\mathcal{K}:=\left\{x \in \mathbb{C}^{16} \mid \Phi_{1}(\widetilde{u}, \widetilde{v})=\right.$ $\left.0, \Phi_{2}(\widetilde{u}, \widetilde{v})=0\right\}$. Let $x \in \mathcal{M}$. Below we introduce some coordinates in a neighborhood of $x$ on $\mathcal{K}$.

For any $z \in \mathbb{C}^{4}$ such that $z \neq 0$ we define the unit vector $g(z)=z /\|z\| \in \mathbb{C}^{4}$. Let $\varphi_{i}, i=1, \ldots, 7$ be some coordinate system on $S^{7}$ (embedded in $\mathbb{C}^{8}$ as a unit sphere with the origin at zero) in some neighborhood $V_{u}$ of $g\left(\tilde{u}_{2}(x)\right)$ and $\psi_{i}, i=1, \ldots, 7$ be some coordinate system on $S^{7}$ in some neighborhood $V_{v}$ of $g\left(\tilde{v}_{1}(x)\right)$. We will use the following functions defined in some neighborhood of $x$ on $\mathcal{K}(z \in \mathcal{K})$ :

$$
\begin{array}{ll}
\tilde{\varphi}_{i}(z)=\varphi_{i} \circ g \circ \tilde{u}_{2}(z), & i=1, \ldots, 7 \\
\tilde{\psi}_{i}(z)=\psi_{i} \circ g \circ \tilde{v}_{1}(z), & i=1, \ldots, 7 .
\end{array}
$$

Let $T_{z} S^{7}$ be the maximal complex subspace of the tangent space of $S^{7}$. For each $z \in V_{u}$ let $x_{1}, \ldots, x_{6}$ be coordinates on $T_{z} S^{7}$ and for each $z \in V_{v} y_{1}, \ldots, y_{6}$ be coordinates on $T_{z} S^{7}$.

Let $\tilde{x}_{1}, \ldots, \tilde{x}_{6}$ and $\tilde{y}_{1}, \ldots, \tilde{y}_{6}$ be functions on $\mathcal{K}$ defined as follows. 
Let $z=\left(\tilde{u}_{1}, \tilde{u}_{2}, \tilde{v}_{1}, \tilde{v}_{2}\right) \in \mathcal{K}$ be in a small enough neighborhood of $x$. By definition $\operatorname{Pr}_{u}$ is the projection from $\mathbb{C}^{4}$ to $T_{g\left(\tilde{u}_{2}\right)} S^{7}$ and $\operatorname{Pr}_{v}$ is the projection from $\mathbb{C}^{4}$ to $T_{g\left(\tilde{v}_{1}\right)} S^{7}$. By definition

$$
\begin{array}{ll}
\tilde{x}_{i}=x_{i} \circ \operatorname{Pr}_{u} \circ \widetilde{u}_{1}, & i=1, \ldots, 6, \\
\tilde{y}_{i}=y_{i} \circ \operatorname{Pr}_{v} \circ \widetilde{v}_{2}, & i=1, \ldots, 6 .
\end{array}
$$

Now let $\operatorname{Pr}_{u}^{\prime}$ and $\operatorname{Pr}_{v}^{\prime}$ be the complex-valued functions defined on $\mathbb{C}^{4}$ by the formulas

$$
\begin{array}{ll}
\operatorname{Pr}^{\prime}{ }_{u}(f)=\left\langle g\left(\widetilde{u}_{2}\right), f\right\rangle, & f \in \mathbb{C}^{4} \\
\operatorname{Pr}^{\prime}{ }_{v}(f)=\left\langle g\left(\widetilde{v}_{1}\right), f\right\rangle, & f \in \mathbb{C}^{4} .
\end{array}
$$

By definition

$$
p:=\operatorname{Pr}_{u}^{\prime} \circ \widetilde{u}_{1}, \quad q:=\operatorname{Pr}_{v}^{\prime} \circ \widetilde{v}_{2} .
$$

Thus, the functions $\tilde{\varphi}_{i}, \tilde{\psi}_{i}, \tilde{x}_{k}, \tilde{y}_{l}, p, q$, where $i, j=1, \ldots, 7$ and $k, l=1, \ldots, 6$, are coordinates on $\mathcal{K}$ in some neighborhood of the point $x$. Locally the manifold $\mathcal{M}$ is a sub-manifold of $\mathcal{K}$ defined by the constraint $\Phi_{3}=0$. In our coordinates this constraint has a form

$$
p\left(1-\sum_{i=1}^{6} y_{i}^{2}-|q|^{2}\right)^{\frac{1}{2}}+q\left(1-\sum_{i=1}^{6} x_{i}^{2}-|p|^{2}\right)^{\frac{1}{2}}=0 .
$$

Therefore $\tilde{\varphi}_{i}, \tilde{\psi}_{i}, \tilde{x}_{k}, \tilde{y}_{l}, p$, where $i, j=1, \ldots, 7$ and $k, l=1, \ldots, 6$ are the coordinates on $\mathcal{M}$ in some neighborhood of $x$. The second differential of $J$ at the point $x$ in this coordinates has the form

$$
\mathrm{d}^{2} J=\lambda_{+} \sum_{i=1}^{6} \mathrm{~d} x_{i}^{2}-\lambda_{-} \sum_{i=1}^{6} \mathrm{~d} y_{i}^{2}+\left(\lambda_{+}-\lambda_{-}\right)|\mathrm{d} p|^{2} .
$$

Since $\lambda_{+}-\lambda_{-}=\|\mathbf{w}\|>0$ for the present case, the Morse indices of this point are $\nu_{+}=$ $8, \nu_{-}=6$ (note that $p$ is a complex coordinate).

Similar treatment of the region $\mathcal{O}_{4}=\left\{x \in \mathcal{M} \mid \tilde{u}_{1} \neq 0, \tilde{v}_{2} \neq 0\right\}$ shows the existence of the critical sub-manifold $\mathcal{M}_{+}^{\mathbf{w}}=\left\{x \in \mathcal{M} \mid \tilde{u}_{2}=\tilde{v}_{1}=0\right\}$. This sub-manifold corresponds to the critical value $\left.J\right|_{\mathcal{M}_{+}^{w}}=\lambda_{+}$and its Morse indices are $\nu_{+}=6, \nu_{-}=8$. Since $\bigcup_{i=0}^{4} \mathcal{O}_{i}=\mathcal{M}$, this concludes the proof for the case $0<\|\mathbf{w}\|<1$.

Case 1. $\mathbf{w}=0$. Consider in $\mathcal{M}$ the open subset $\mathcal{O}_{1}$.

Let $\eta_{3}=0$. Then in the region $\mathcal{O}_{1}$ Eqs. (14) and (15) imply that $\eta_{1} \tilde{v}_{1}=\eta_{2} \tilde{v}_{2}=0 \Rightarrow \eta_{1}=$ $\eta_{2}=0$. Equations (12) and (13) for such $\eta_{i}$ have only the solution $\tilde{u}_{1}=\tilde{u}_{2}=0$ which defines the global minimum manifold $\mathcal{M}_{\min }^{(0,0,0)}=\left\{x \in \mathcal{M} \mid \tilde{u}_{1}=\tilde{u}_{2}=0\right\}$. Now let $\eta_{3} \neq 0$ and $\tilde{u}_{1} \neq 0$ or $\tilde{u}_{2} \neq 0$. In this case Eqs. (12)- 15$)$ give $\left|\eta_{3}\right|^{2}=\left(1+\eta_{1}\right)\left(1+\eta_{2}\right)$ and $\left|\eta_{3}\right|^{2}=\eta_{1} \eta_{2}$, which imply $\eta_{2}=-1-\eta_{1}$ and $\left|\eta_{3}\right|^{2}=-\eta_{1}\left(1+\eta_{1}\right)$. Then Eqs. (12) and (15) have the solution

$$
\tilde{u}_{2}=-\frac{1+\eta_{1}}{\eta_{3}} \tilde{u}_{1}=z \tilde{u}_{1}, \quad \tilde{v}_{1}=-\frac{\eta_{2}}{\eta_{3}^{*}} \tilde{v}_{2}=-z^{*} \tilde{v}_{2}
$$


where we used the notation $z=-\left(1+\eta_{1}\right) / \eta_{3} \in \mathbb{C} /\{0\}$ and the relation $-\eta_{2} / \eta_{3}^{*}=-z^{*}$. Note that for a given pair $\left(\tilde{u}_{1}, \tilde{v}_{2}\right) \in \mathbb{C}^{8}$, $z$ can be any non-zero complex number such that $\left(\tilde{u}_{1}, z \tilde{u}_{1},-z^{*} \tilde{v}_{2}, \tilde{v}_{2}\right) \in \mathcal{M}$. The solutions of the form (20) constitute the critical set $\mathcal{T}=\left\{x \in \mathcal{O}_{1} \mid \tilde{u}_{2}=z \tilde{u}_{1}, \tilde{v}_{1}=-z^{*} \tilde{v}_{2}, z \in \mathbb{C}\right\} \subset \mathcal{M}_{\text {saddle }}^{(0,0,0)}$. A similar treatment of the region $\mathcal{O}_{2}$ shows that the objective function in this region has as critical points only the global maximum manifold $\mathcal{M}_{\max }^{(0,0,0)}=\left\{x \in \mathcal{M} \mid \tilde{v}_{1}=\tilde{v}_{2}=0\right\}$ and the set $\mathcal{T}$.

Now consider the region $\mathcal{O}_{3}$.

Let $\eta_{3}=0$. Then in the region $\mathcal{O}_{3}$ Eqs. (13) and (14) imply $\left(1+\eta_{2}\right) \tilde{u}_{2}=\eta_{1} \tilde{v}_{1}=0 \Rightarrow$ $\eta_{1}=0, \eta_{2}=-1$. The solution of Eqs. (12) and (15) for such values of $\eta_{i}$ gives the critical set $\left\{x \in \mathcal{M} \mid \tilde{u}_{1}=\tilde{v}_{2}=0\right\} \subset \mathcal{M}_{\text {saddle }}^{(0,0,0)}$.

Let $\eta_{3} \neq 0$. The treatment is similar to the treatment of the case $\eta_{3} \neq 0$ for the region $\mathcal{O}_{1}$ and gives the critical set $\mathcal{T}$. A similar treatment of the region $\mathcal{O}_{4}$ shows that the set of critical points of the objective function in this region is $\left\{x \in \mathcal{M} \mid \tilde{u}_{2}=\tilde{v}_{1}=0\right\} \bigcup \mathcal{T}$.

Combining together the results for the regions $\mathcal{O}_{1}, \mathcal{O}_{2}, \mathcal{O}_{3}$, and $\mathcal{O}_{4}$, we find that the critical manifolds are the global minimum manifold $\mathcal{M}_{\min }^{(0,0,0)}$, the global maximum manifold $\mathcal{M}_{\text {max }}^{(0,0,0)}$, and the set $\mathcal{T} \bigcup\left\{x \in \mathcal{M} \mid \tilde{u}_{2}=\tilde{v}_{1}=0\right\} \bigcup\left\{x \in \mathcal{M} \mid \tilde{u}_{1}=\tilde{v}_{2}=0\right\} \equiv \mathcal{M}_{\text {saddle }}^{(0,0,0)}$. Since 4 $\bigcup_{i=1} \mathcal{O}_{i}=\mathcal{M}$, these manifolds are all critical manifolds of the objective function $J$ for the case $\mathbf{w}=0$. A simple computation using the constraints (5)-(7) shows that the value of the objective function at any point $x \in \mathcal{M}_{\text {saddle }}^{(0,0,0)}$ equals to $1 / 2$, i.e., $\left.J\right|_{\mathcal{M}^{0}}=1 / 2$.

Now we will find Morse indices of the critical manifold $\mathcal{M}_{\text {saddle }}^{(0,0,0)}$. An arbitrary point $x=\left(u_{1}, u_{2}, v_{1}, v_{2}\right) \in \mathcal{M}_{\text {saddle }}^{(0,0,0)}$ can be moved into the point $\tilde{x}=\left(\tilde{u}_{1}, \tilde{u}_{2}, \tilde{v}_{1}, \tilde{v}_{2}\right) \in \mathcal{M}_{\text {saddle }}^{(0,0,0)}$ with $\tilde{u}_{1}=0, \tilde{v}_{2}=0$ by the following transformation:

$$
\begin{aligned}
& \tilde{u}_{1}=\alpha u_{1}+\beta u_{2}, \quad \tilde{u}_{2}=-\beta^{*} u_{1}+\alpha^{*} u_{2}, \\
& \tilde{v}_{1}=\alpha v_{1}+\beta v_{2},
\end{aligned}
$$

where $\alpha, \beta \in \mathbb{C},|\alpha|^{2}+|\beta|^{2}=1$. For example, $\alpha=-\beta z$ for $x=\left(u_{1}, z u_{1},-z^{*} v_{2}, v_{2}\right) \in \mathcal{T}$.

As in the analysis of the Morse indices for the case 2 , in some neighborhood of $\tilde{x}$ we can introduce the coordinates $\tilde{\varphi}_{i}, \tilde{\psi}_{i}, \tilde{x}_{k}, \tilde{y}_{l}, p, q$, where $i, j=1, \ldots, 7$ and $k, l=1, \ldots, 6$. These coordinates satisfy the constraint:

$$
p\left(1-\sum_{i=1}^{6} \tilde{y}_{i}^{2}-|q|^{2}\right)^{\frac{1}{2}}+q\left(1-\sum_{i=1}^{6} \tilde{x}_{i}^{2}-|p|^{2}\right)^{\frac{1}{2}}=0 .
$$

The second differential of $J$ in these coordinates has the form:

$$
\mathrm{d}^{2} J=\sum_{i=1}^{6} \mathrm{~d} \tilde{x}_{i}^{2}-\sum_{i=1}^{6} \mathrm{~d} \tilde{y}_{i}^{2}+0 \cdot|\mathrm{d} p|^{2} .
$$

It is easy to see that the tangent space to $\mathcal{M}_{\text {saddle }}^{(0,0,0)}$ at the point $\tilde{x}$ is spanned by the vectors

$$
\frac{\partial}{\partial \tilde{\varphi}_{i}}, \frac{\partial}{\partial \tilde{\psi}_{i}}, \frac{\partial}{\partial \operatorname{Re} p}, \frac{\partial}{\partial \operatorname{Im} p}
$$


Therefore $\mathcal{M}_{\text {saddle }}^{(0,0,0)}$ is nondegenerate, $\operatorname{dim} \mathcal{M}_{\text {saddle }}^{(0,0,0)}=16$ and the Morse indices of $\mathcal{M}_{\text {saddle }}^{(0,0,0)}$ are $\nu_{+}=\nu_{-}=6$.

Case 3. $\|\mathbf{w}\|=1$. In this case $\lambda_{-}=0, \lambda_{+}=1$, and

$$
J\left[\tilde{u}_{1}, \tilde{u}_{2}, \tilde{v}_{1}, \tilde{v}_{2}\right]=\left\|\tilde{u}_{1}\right\|^{2} .
$$

Let $\mathcal{U}_{1}=\left\{x \in \mathcal{M} \mid \tilde{v}_{1} \neq 0\right\}$. Clearly, points in $\mathcal{U}_{1}$ with $\tilde{u}_{1}=0$ form the global minimum of the objective. Assume that there are critical points in $\mathcal{U}_{1}$ such that $\tilde{u}_{1} \neq 0$. For such points Eqs. (12)-(15) imply the system of equations

$$
\begin{aligned}
& \left|\eta_{3}\right|^{2}=\eta_{1} \eta_{2} \\
& \left|\eta_{3}\right|^{2}=\eta_{2}\left(1+\eta_{1}\right)
\end{aligned}
$$

which has only the solutions with $\eta_{2}=\eta_{3}=0$. But in the region $\mathcal{U}_{1}, \tilde{v}_{1} \neq 0$ and therefore Eq. (14) implies $\eta_{1}=0$. Then, Eq. (12) for $\eta_{1}=\eta_{2}=\eta_{3}=0$ has the solution $\tilde{u}_{1}=0$ which contradicts the assumption $\tilde{u}_{1} \neq 0$. As a result, the only critical points in $\mathcal{U}_{1}$ are with $\tilde{u}_{1}=0$. These points form the global minimum manifold $\mathcal{M}_{\text {min }}^{\mathbf{w}}=\left\{x \in \mathcal{M} \mid \tilde{u}_{1}=0\right\}$. This manifold is diffeomorphic to the space bundle with $S^{7}$ as a base and $S^{14}$ as a fibre. Thus, $\mathcal{M}_{\text {min }}^{\mathbf{w}}$ is connected. We can use $\tilde{u}_{1}$ as normal coordinates in some neighborhood of $\mathcal{M}_{\text {min }}^{\mathbf{w}}$. Thus $\mathcal{M}_{\min }^{\mathrm{w}}$ is nondegenerate.

The treatment of the region $\mathcal{U}_{2}=\left\{x \in \mathcal{M} \mid \tilde{u}_{1} \neq 0\right\}$ is equivalent to the previous consideration. The critical points in this region form the global maximum manifold $\mathcal{M}_{\max }^{\mathbf{w}}=$ $\left\{x \in \mathcal{M} \mid \tilde{v}_{1}=0\right\}$. Note that $\mathcal{U}_{1} \cup \mathcal{U}_{2}=\mathcal{M}$. Therefore, all critical points of $J$ correspond to the global minimum $J=0$ and global maximum $J=1$. The critical manifolds corresponding to the minimum and the maximum are connected and nondegenerate.

Remark 1 The critical manifolds in terms of the original parametrization of the Kraus operators by $\left(u_{1}, u_{2}, v_{1}, v_{2}\right)$ can be obtained by expressing $\tilde{u}_{i}$ and $\tilde{v}_{i}$ in terms of $u_{i}$ and $v_{i}$. If $z_{0} \neq 0$, then it follows from (8) and (9) that

$$
\begin{array}{ll}
\tilde{u}_{1}=\mu u_{1}+\frac{z_{0}}{\left|z_{0}\right|} \nu u_{2}, & \tilde{u}_{2}=-\nu u_{1}+\frac{z_{0}}{\left|z_{0}\right|} \mu u_{2} \\
\tilde{v}_{1}=\mu v_{1}+\frac{z_{0}}{\left|z_{0}\right|} \nu v_{2}, & \tilde{v}_{2}=-\nu v_{1}+\frac{z_{0}}{\left|z_{0}\right|} \mu v_{2}
\end{array}
$$

Thus, for $z_{0} \neq 0$ and $0<\|\mathbf{w}\|<1$ the critical manifolds are the following: the global minimum $\mathcal{M}_{\min }^{\mathbf{w}}=\left\{x \in \mathcal{M} \mid u_{1}=u_{2}=0\right\}$, the global maximum $\mathcal{M}_{\max }^{\mathbf{w}}=\left\{x \in \mathcal{M} \mid v_{1}=\right.$ $\left.v_{2}=0\right\}$, and the saddles $\mathcal{M}_{ \pm}^{\mathbf{w}}=\left\{x \in \mathcal{M} \mid u_{2}=z_{ \pm} u_{1}, v_{1}=-z_{ \pm}^{*} v_{2}\right\}$. Here $z_{ \pm}=z_{0}^{*} /(\gamma \pm\|\mathbf{w}\|)$. For $z_{0} \neq 0$ and $\|\mathbf{w}\|=1$ (hence $\gamma \neq 1$ ), the critical manifolds are $\mathcal{M}_{\text {min }}^{\mathbf{w}}=\left\{x \in \mathcal{M} \mid u_{2}=\right.$ $\left.z_{0}^{*} u_{1} /(\gamma-1)\right\}, \mathcal{M}_{\max }^{\mathbf{w}}=\left\{x \in \mathcal{M} \mid v_{2}=z_{0}^{*} v_{1} /(\gamma-1)\right\}$, and there are no saddles.

If $z_{0}=0$ and $\gamma \geq 0$, then $\tilde{u}_{1}=u_{1}, \tilde{u}_{2}=u_{2}, \tilde{v}_{1}=v_{1}$, and $\tilde{v}_{2}=v_{2}$. Thus for $\gamma=0$, $\mathcal{M}_{\text {min }}^{(0,0,0)}=\left\{x \in \mathcal{M} \mid u_{1}=u_{2}=0\right\}, \mathcal{M}_{\max }^{(0,0,0)}=\left\{x \in \mathcal{M} \mid v_{1}=v_{2}=0\right\}$, and $\mathcal{M}_{\text {saddle }}^{(0,0,0)}=\{x \in$ $\left.\mathcal{M} \mid u_{2}=z u_{1}, v_{1}=-z^{*} v_{2}, z \in \mathbb{C}\right\} \bigcup\left\{x \in \mathcal{M} \mid u_{1}=v_{2}=0\right\}$. For $0<\gamma<1$ the critical manifolds are $\mathcal{M}_{\min }^{(0,0, \gamma)}=\left\{x \in \mathcal{M} \mid u_{1}=u_{2}=0\right\}, \mathcal{M}_{\max }^{(0,0, \gamma)}=\left\{x \in \mathcal{M} \mid v_{1}=v_{2}=0\right\}$, and the saddles $\mathcal{M}_{-}^{(0,0, \gamma)}=\left\{x \in \mathcal{M} \mid u_{1}=v_{2}=0\right\}$ and $\mathcal{M}_{+}^{(0,0, \gamma)}=\left\{x \in \mathcal{M} \mid u_{2}=v_{1}=0\right\}$. For $\gamma=1$, $\mathcal{M}_{\min }^{(0,0,1)}=\left\{x \in \mathcal{M} \mid u_{1}=0\right\}$ and $\mathcal{M}_{\max }^{(0,0,1)}=\left\{x \in \mathcal{M} \mid v_{1}=0\right\}$. 
If $z_{0}=0$ and $\gamma<0$, then $\tilde{u}_{1}=u_{2}, \tilde{u}_{2}=u_{1}, \tilde{v}_{1}=v_{2}$, and $\tilde{v}_{2}=v_{1}$. In this case for $-1<\gamma<0$ the critical manifolds are the following: $\mathcal{M}_{\min }^{(0,0, \gamma)}=\left\{x \in \mathcal{M} \mid u_{1}=u_{2}=0\right\}$, $\mathcal{M}_{\max }^{(0,0, \gamma)}=\left\{x \in \mathcal{M} \mid v_{1}=v_{2}=0\right\}, \mathcal{M}_{-}^{(0,0, \gamma)}=\left\{x \in \mathcal{M} \mid u_{2}=v_{1}=0\right\}$ and $\mathcal{M}_{+}^{(0,0, \gamma)}=\{x \in$ $\left.\mathcal{M} \mid u_{1}=v_{2}=0\right\}$. For $\gamma=-1, \mathcal{M}_{\min }^{(0,0,-1)}=\left\{x \in \mathcal{M} \mid u_{2}=0\right\}$ and $\mathcal{M}_{\max }^{(0,0,-1)}=\left\{x \in \mathcal{M} \mid v_{2}=\right.$ $0\}$.

Remark 2 The values of the objective function at the saddle points satisfy the equality $J_{+}(\mathbf{w})+J_{-}(\mathbf{w})=1$. This fact is a consequence of the more general symmetry of the objective function, defined by the duality map $T: \mathcal{M} \rightarrow \mathcal{M}$ such that $T\left(u_{1}, u_{2}, v_{1}, v_{2}\right)=\left(v_{1}, v_{2}, u_{1}, u_{2}\right)$ as $J[x ; \mathbf{w}]+J[T(x) ; \mathbf{w}]=1$ for any $x \in \mathcal{M}$. Thus, if the level set $\Gamma_{\mathbf{w}}(\alpha):=\{x \in \mathcal{M} \mid J[x, \mathbf{w}]=$ $\alpha\}$ for some value $\alpha \in[0,1]$ is known then one immediately gets the level set for the value $1-\alpha$ as $\Gamma_{\mathbf{w}}(1-\alpha)=T\left(\Gamma_{\mathbf{w}}(\alpha)\right)$.

\section{Connectivity of the level sets}

The level set $\Gamma_{\mathbf{w}}(\mu)$ for an admissible objective value $\mu \in[0,1]$ is defined as the set of all controls $x=\left(u_{1}, u_{2}, v_{1}, v_{2}\right) \in \mathcal{M}$ which produce the same outcome value $\mu$ for the objective function $J\left[u_{1}, u_{2}, v_{1}, v_{2} ; \mathbf{w}\right]$, i.e., $\Gamma_{\mathbf{w}}(\mu)=\{x \in \mathcal{M} \mid J[x ; \mathbf{w}]=\mu\}$ (we omit the subscript $\mathbf{w}$ in the sequel). In this section it is shown that each level set for the function $J[\cdot ; \mathbf{w}]$ is connected. This means that any pair of solutions in a level set $\Gamma(\mu)$ is connected via a continuous pathway of solutions entirely passing through $\Gamma(\mu)$. Practically, connectivity of the level sets implies the possibility to experimentally locate more desirable solutions via continuous variations of the control parameters while maintaining the same value of the objective function. The proof of the connectivity of the level sets for the objective functions defined by (4) is based on generalized Morse theory, which is presented in the remainder of this section. Theorem 2 below formulates the conditions for a generalized Morse function to have connected level sets. These conditions are satisfied for the objective function $J[\cdot, \mathbf{w}]$ defined by (4), as stated in the end of this section. Formulation of Theorem 2 includes a very general class of functions and can be applied to the investigation of connectivity of the level sets for situations beyond the scope of this paper, including landscapes for multilevel closed and open systems.

\subsection{Connectivity of level sets of generalized Morse functions}

Let $M$ be a smooth compact manifold of dimension $d$, and let $f$ be a smooth function $f: M \rightarrow \mathbb{R}$. We suppose that the critical set of $f, S:=\{x \in M \mid \mathrm{d} f(x)=0\}$ is a disjoint union of smooth connected sub-manifolds $C_{i}(i=1,2, \ldots, n)$ of dimension $d_{i}$. Let $\mu_{i}=\left.f\right|_{C_{i}}$.

For each point $x \in C_{i}$ there exists an open neighborhood $U$ of $x$ and a coordinate system $\left\{x_{l}\right\}$ in $U$ such that

$$
C_{i} \cap U=\left\{x \in U \mid x_{d_{i}+1}=\cdots=x_{n}=0\right\} .
$$

Consider the following matrix

$$
J_{i}(x):=\left\|\frac{\partial^{2} f(x)}{\partial x_{l} \partial x_{m}}\right\|_{l, m=d_{i}+1, \ldots, d}, \quad x \in C_{i} .
$$


It is easy to see that if $\left\{y_{l}\right\}$ is another coordinate system in $U$ such that

$$
C_{i} \cap U=\left\{y \in U \mid y_{d_{i}+1}=\cdots=y_{n}=0\right\},
$$

and

$$
\widetilde{J}_{i}(x):=\left\|\frac{\partial^{2} f(x)}{\partial y_{l} \partial y_{m}}\right\|_{l, m=d_{i}+1, \ldots, d}, \quad x \in C_{i}
$$

then

$$
\operatorname{rank} J_{i}(x)=\operatorname{rank} \widetilde{J}_{i}(x)
$$

Therefore we can give the following

Definition 2 The point $x \in C_{i}$ is said to be nondegenerate if $\operatorname{det} J_{i}(x) \neq 0$.

Definition $3 A$ critical sub-manifold $C_{i}$ is said to be nondegenerate if $\forall x \in C_{i}, x$ is a nondegenerate point.

Let $x \in C_{i}$ and $\lambda_{i}^{+}(x), \lambda_{i}^{-}(x)$ be the numbers of positive and negative eigenvalues of the matrix $J_{i}(x)$. It is clear that $\lambda_{i}^{+}(x), \lambda_{i}^{-}(x)$ do not depend on the choice of coordinate system $\left\{x_{i}\right\}$ in the neighborhood of $x$. One can prove that $\lambda_{i}^{+}(x)$ and $\lambda_{i}^{-}(x)$ do not depend on the point $x \in C_{i}\left(\lambda_{i}^{+}(x)\right.$ and $\lambda_{i}^{-}(x)$ are continuous and $C_{i}$ is connected.). Let $\lambda_{i}^{+}:=\lambda_{i}^{+}(x)$ and $\lambda_{i}^{-}:=\lambda_{i}^{-}(x) . \lambda_{i}^{+}$and $\lambda_{i}^{-}$are called the indices of $C_{i}$.

Definition 4 Let $M$ be a smooth compact connected manifold and $f: M \rightarrow \mathbb{R}$. Suppose that the critical set of $f$ is a disjoint union of (compact) connected nondegenerate sub-manifolds $C_{i}$. In this case we say that $f$ is a generalized Morse function. Sub-manifolds $C_{i}$ are called the critical sub-manifolds of $f$.

Theorem 2 Let $M$ be a smooth compact connected manifold and $f$ be a generalized Morse function. Let $C_{i}, i=1, \ldots, n$ be critical sub-manifolds of $f$ and $\mu_{i}=\left.f\right|_{C_{i}}$. We can assume that $\mu_{\min }:=\mu_{1} \leq \mu_{2} \leq \ldots \leq \mu_{n}=: \mu_{\max }$. Suppose that the sub-manifold $C_{\max }:=f^{-1}\left(\mu_{\max }\right)$ is connected. Suppose also that $\forall i=1, \ldots, n-1$ the indices $\lambda_{i}^{+} \geq 2, \lambda_{i}^{-} \geq 2$. Then $\forall \mu: \mu_{\min } \leq \mu \leq \mu_{\max }$ the set $\Gamma(\mu):=f^{-1}(\mu)$ is connected.

Proof. We decompose the proof of the theorem into a sequence of several Lemmas.

Lemma 1 There exists an open neighborhood $U$ of $C_{\max }$ such that $U$ is diffeomorphic to some bundle $\mathcal{E}$ with the base $C_{\max }$ and the fibre $B_{d-d_{n}}$. Here $B_{k}$ is a $k$-dimensional ball.

Proof. $\mathrm{M}$ is a compact. Therefore there exists a Riemann metric $g \in \operatorname{sym}\left(T^{*} M \otimes T^{*} M\right)$. (Here $T^{*} M$ is a cotangent bundle of $M$.) By definition, $\mathcal{L}$ is a restriction of the tangent bundle $T M$ to $C_{\max }$. Let $\mathcal{N}$ be a sub-bundle of $\mathcal{L}$ such that $\forall x \in C_{\max }$ the fiber $\mathcal{N}_{x}$ of $\mathcal{N}$ over $x$ is a subspace of $T_{x} M$ consisting of all vectors orthogonal to $T_{x} C_{\max }$. Let $\mathcal{B}_{l}$ be a sub-bundle of $\mathcal{N}$ such that $\forall x \in C_{\max }$ the fiber $\left(\mathcal{B}_{l}\right)_{x}$ of $\mathcal{B}_{l}$ is a set of all vectors $v$ of $\mathcal{N}_{x}$ satisfying the following inequality: $\|v\|<l$ (with respect to the metric $g$ ). 
Let $\gamma_{v}(x)(t)\left(x \in M, v \in T_{x} M, t \in \mathbb{R}\right)$ be a geodesic line, i.e., the solution of the following ordinary differential equation

$$
\nabla_{\dot{\gamma}_{v}(x)(t)} \dot{\gamma}_{v}(x)(t)=0
$$

with the following initial conditions

$$
\begin{aligned}
\gamma_{v}(x)(0) & =x \\
\left.\dot{\gamma}_{v}(x)(t)\right|_{t=0} & =v .
\end{aligned}
$$

Here $\nabla_{v}$ is a Levi-Civita connection on $M$ with respect to the metric $g$. The solution of this differential equation is defined on the whole real line because $M$ is compact.

Let $F_{l}$ for $l \in(0,+\infty)$ be a map $\mathcal{B}_{l} \rightarrow M$ which assigns to each point $(x, v) \in \mathcal{B}_{l}$ $\left(x \in C_{\max }, v \in\left(\mathcal{B}_{l}\right)_{x}\right)$ the point $\gamma_{v}(x)(1)$. It follows from the inverse function theorem that there exits a number $l_{0}>0$ such that $F_{l}$ is a diffeomorphism on its image for all $l: 0<l \leq l_{0}$.

Lemma 2 If $\varepsilon$ is small enough then $\forall \mu: \mu_{\max }>\mu>\mu_{\max }-\varepsilon$ the set $\Gamma(\mu)=f^{-1}(\mu)$ is connected.

Proof. Let $l_{0}$ be a number from the previous Lemma. It follows from the Morse Lemma that for every $x \in C_{\max }$ we can choose coordinates $z_{1}, \ldots, z_{d-d_{n}}$ on $\left(\mathcal{B}_{l_{0}}\right)_{x}$ in some neighborhood $U$ of zero such that

$$
\left.f \circ F_{l_{0}}\right|_{U}=z_{1}^{2}+\ldots+z_{d-d_{n}}^{2}
$$

Moreover, from construction of these coordinates it follows that in some neighborhood of every point $x_{0} \in C_{\max }$ they are differentiable functions of $x$. Therefore, there exists a finite covering $\left\{U_{i}\right\}_{i=1, \ldots, q}$ of $C_{\max }$ by open connected sets and a family of diffeomorphisms $g_{i}: U_{i} \times B_{d-d_{n}} \rightarrow \pi^{-1}\left(U_{i}\right)(i=1, \ldots, q)$ on its image commuting with the projections such that

$$
f \circ F_{l_{0}} \circ g_{i}=z_{1}^{2}+\ldots+z_{d-d_{n}}^{2}, \quad i=1, \ldots, q .
$$

Here $z_{i}, i=1, \ldots, d-d_{n}$ are some coordinates on the ball $B_{d-d_{n}}$ and $\pi$ is a canonical projection from $\mathcal{B}_{l_{0}}$ to $C_{\text {max }}$.

We now prove that for every $l_{1}: 0<l_{1}<l_{0}$ there exists $\varepsilon_{1}>0$ such that $\forall \mu: \mu_{\max }-\varepsilon_{1}<$ $\mu \leq \mu_{\max }, \Gamma(\mu) \subset F_{l_{1}} \mathcal{B}_{l_{1}}$. Suppose that $\forall n=1,2, \ldots$ there exists a point $x_{n}$ such that $f\left(x_{n}\right)>\mu_{\max }-1 / n$ and $x_{n} \notin F_{l_{1}} \mathcal{B}_{l_{1}}$. Because $M \backslash F_{l_{1}} \mathcal{B}_{l_{1}}$ is compact, then there exists a point $x_{0} \in M \backslash F_{l_{1}} \mathcal{B}_{l 1}$ and sub-sequence $\left\{x_{n_{k}}\right\}$ of $\left\{x_{n}\right\}$ such that $x_{n_{k}} \rightarrow x_{0}$ as $k \rightarrow \infty$. We find that $f\left(x_{0}\right)=\mu_{\max }$ and $x_{0} \in C_{\max }$. This contradiction proves our statement. If $l_{1}$ is small enough then $\mathcal{B}_{l_{1}} \cap U_{i} \subset g_{i}\left(U_{i} \times B_{d-d_{n}}\right)$ for all $i=1, \ldots, q$. Therefore if $\mu>\mu_{\max }-\varepsilon_{1}$ then $f^{-1}(\mu) \cap \pi^{-1}\left(U_{i}\right) \subset g_{i}\left(U_{i} \times B_{d-d_{n}}\right)$ and connected. So we find that $f^{-1}(\mu)$ is connected if $\mu>\mu_{\max }-\varepsilon_{1}$.

Lemma 3 Suppose that for some $\mu: \mu_{i}<\mu<\mu_{i+1}(i=1, \ldots, n-1)$ the set $\Gamma(\mu)$ is connected. Then $\forall \mu$ such that $\mu_{i}<\mu<\mu_{i+1}$, the set $\Gamma(\mu)$ is connected. 
Proof. Let $\nu \in \mathbb{R}: \mu_{i}<\nu<\mu_{i+1}$. Let us prove that $\Gamma(\nu)$ is connected. We can assume that $\nu<\mu$, and let $\varepsilon$ be a positive number such that $\mu_{i}<\nu-\varepsilon<\mu+\varepsilon<\mu_{i+1}$. Consider the following sets

$$
\begin{aligned}
U_{\varepsilon} & =\{x \mid \nu-\varepsilon<f(x)<\mu+\varepsilon\} \\
\bar{U}_{\varepsilon} & =\{x \mid \nu-\varepsilon \leq f(x) \leq \mu+\varepsilon\}
\end{aligned}
$$

Consider also the following differential equation on $M$

$$
\dot{\gamma}(t)=\frac{\operatorname{grad} f(\gamma(t))}{\|\operatorname{grad} f(\gamma(t))\|^{2}} .
$$

(Recall that $M$ has a Riemann metric). The right hand side of this equation is well defined on $U_{\varepsilon}$. The solution of (35) is $\gamma_{x}(t)$ with the initial condition

$$
\gamma_{x}(0)=x, \quad x \in \Gamma(\mu) .
$$

By the extension theorem [49, 50] this solution must leave the compact set $\bar{U}_{\varepsilon / 2}$. It is easy to prove that $f\left(\gamma_{x}(t)\right)=t+\mu$. So the solution $\gamma_{x}(t)$ is defined and unique on the interval $(\nu-\mu-\varepsilon / 3, \mu+\varepsilon / 3)$. Therefore we have a smooth map $\Delta_{\mu, \nu}: \Gamma(\mu) \rightarrow \Gamma(\nu), x \mapsto \gamma_{x}(\nu-\mu)$. By the same means we can construct the map $\Delta_{\nu, \mu}: \Gamma(\nu) \rightarrow \Gamma(\mu) . \Delta_{\mu, \nu}(x)=y$ if and only if $x$ and $y$ lie on the same integral curve of (35). We have $\Delta_{\mu, \nu} \circ \Delta_{\nu, \mu}=\mathrm{id}$ and $\Delta_{\nu, \mu} \circ \Delta_{\mu, \nu}=\mathrm{id}$. So $\Gamma(\mu)$ and $\Gamma(\nu)$ are diffeomorphic.

Lemma 4 Suppose that the assumptions of the theorem hold. Let $\mu \in \mathbb{R}: \mu_{i}<\mu<\mu_{i+1}$, $\mu_{i}=2, \ldots, n-1$, and $\Gamma(\mu)$ is connected. Then $\forall \nu$ such that $\mu_{i-1}<\nu<\mu_{i}$, the set $\Gamma(\nu)$ is also connected.

Proof. We prove this lemma only for the case of connected $C_{i}$. The general case is analogous to this case.

As in Lemma 1, let $\mathcal{B}_{l}$ be a bundle with the base $C_{i}$ which consists of all vectors $v$ normal to $C_{i}$ and such that $\|v\|<l$. We have $\mathcal{B}_{l_{1}} \subset \mathcal{B}_{l_{2}}$ for $l_{1}<l_{2}$. Let $F_{l}$ be a map $\mathcal{B}_{l} \rightarrow M$ constructed as in Lemma 1. As in Lemma 1, we find that $F_{l}$ is a diffeomorphism if $0<l \leq l_{0}$ for some positive number $l_{0}$. As in Lemma 1 we find that for every $l_{0}^{\prime}<l_{0}$ there exists a covering $\left\{U_{j}\right\}_{j=1, \ldots, p}$ of $C_{i}$ by open connected sets and the family of diffeomorphisms $g_{j}: U_{j} \times B_{d-d_{i}} \rightarrow \pi^{-1}\left(U_{j}\right)$ on its image commuting with the projections such that

$$
f \circ F_{l} \circ g_{j}=z_{1}^{2}+\ldots+z_{\lambda_{i}^{+}}^{2}-z_{\lambda_{i}^{+}+1}^{2}-\ldots-z_{d-d_{i}}^{2}+\mu_{i}
$$

Here $B_{d-d_{i}}$ is a $d-d_{i^{-}}$-dimensional ball and $\pi$ a canonical projection from $\mathcal{B}_{l_{0}^{\prime}}$ to $C_{i}$.

It is easy to see that for every $l_{0}^{\prime}<l_{0}$ there exists a positive number $l_{1}<l_{0}^{\prime}$ such that $\forall j=1, \ldots, p \mathcal{B}_{l_{1}} \cap \pi^{-1}\left(U_{j}\right) \subset g_{j}\left(U_{j} \times B_{d-d_{i}}\right)$. For every $l_{1}<l_{0}^{\prime}$ there exists a positive number $\varepsilon_{2}$ such that $\forall x \in C_{i},\left(\mathcal{B}_{l_{1} / 2}\right)_{x} \cap F_{l_{0}}^{-1}\left(\Gamma\left(\mu_{i}+\kappa\right)\right) \neq \emptyset \forall \kappa:|\kappa|<\varepsilon_{2}$. We now prove that $\mathcal{B}_{l_{1}} \cup \pi^{-1}\left(U_{j}\right) \cap F_{l_{0}}^{-1}(\Gamma(\mu+\kappa))$ is connected $\forall j=1, \ldots, p$ if $|\kappa|<\varepsilon_{2}$. Indeed, let $x_{1}$ and $x_{2}$ be two points which lie in the set $\mathcal{B}_{l_{1}} \cup \pi^{-1}\left(U_{j}\right) \cap F_{l_{0}}^{-1}(\Gamma(\mu+\kappa))$. We can consider only the case $\kappa>0$. The set $g_{j}\left(U_{j} \times B_{d-d_{i}}\right) \cap F_{l_{0}}^{-1}\left(\Gamma\left(\mu_{i}+\kappa\right)\right)$ is diffeomorphic to $\mathbb{R}^{\lambda_{i}^{+}} \times S^{\lambda_{i}^{-}-1} \times U_{i}$ 
and connected. Let $\gamma(t) t \in[0,1]$ be a path in $g_{j}\left(U_{j} \times B_{d-d_{i}}\right) \cap F_{l_{0}}^{-1}\left(\Gamma\left(\mu_{i}+\kappa\right)\right)$ such that $\gamma(0)=x_{1}, \gamma(1)=x_{2}$. Let $d(x)$ be a function on $\mathcal{B}_{l_{0}}$ defined as follows: $d((z, v))=\|v\|^{2}$, where $z \in C_{i}$ and $v \in\left(\mathcal{B}_{l_{0}}\right)_{x}$. Let $x \in F_{l_{0}}^{-1}(\Gamma(\mu+\kappa)) \cap g_{j}\left(U_{j} \times B_{d-d_{i}}\right)$ and $w(x)$ be a projection of $\nabla d(x)$ to the tangent space of $F_{l_{0}}^{-1}(\Gamma(\mu+\kappa))$ at $x$. It is obvious that $w(x) \neq 0$ $\forall x \in F_{l_{0}^{\prime}}^{-1}(\Gamma(\mu+\kappa)) \cap g_{j}\left(U_{j} \times B_{d-d_{i}}\right) \cap\left(\mathcal{B}_{l_{0}^{\prime}} \backslash \mathcal{B}_{l_{1}}\right)$ if $l_{0}^{\prime}$ is a sufficiently small number. So we can retract the path $\gamma(t)$ along the vector field $w$ to the part $\tilde{\gamma}(t)$ which lies in $\mathcal{B}_{l_{1}}$ and connects the points $x_{1}$ and $x_{2}$. So $\mathcal{B}_{l_{1}} \cup \pi^{-1}\left(U_{j}\right) \cap F_{l_{0}}^{-1}(\Gamma(\mu+\kappa))$ is connected. Now we can find that $\mathcal{B}_{l_{1}} \cup \cap F_{l_{0}}^{-1}(\Gamma(\mu+\kappa))$ is connected.

Now let $x_{1}, x_{2} \in \Gamma(\mu), \mu<\mu_{i},\left|\mu-\mu_{i}\right|<\varepsilon_{3}$. Let $U=F_{l_{0}}(\mathcal{B})_{l_{1} / 2}, V=F_{l_{0}}(\mathcal{B})_{l_{1} / 3}$, $W=F_{l_{0}}(\mathcal{B})_{l_{1} / 4}$. At first suppose that $x_{1} \notin U$ and $x_{2} \notin U$. Let $\gamma_{x_{1}}(t), \gamma_{x_{2}}(t)$ be solutions of the differential equation (35) with initial conditions $x_{1}$ and $x_{2}$ respectively. The paths $\gamma_{x_{1}}(t)$ and $\gamma_{x_{2}}(t)$ intersect the sub-manifold $\Gamma\left(\mu+\varepsilon_{3}\right)$ at the points $y_{1}$ and $y_{2}$ if $\varepsilon_{3}$ is enough small. Let $\widetilde{\delta}(t), t \in[0,1]$ be a path such that $\forall t \in[0,1] \widetilde{\delta}(t) \in \Gamma(\mu+\varepsilon)$ and $y_{1}=\widetilde{\delta}(0), y_{2}=\widetilde{\delta}(1)$. We must consider the following two cases.

1) $\widetilde{\delta} \cap V=\emptyset$. If $\varepsilon_{3}$ is small enough then we can deform the part $\widetilde{\delta}$ along the vector field $\nabla f /\|\nabla f\|^{2}$ to the part $\delta$ which lies on $\Gamma(\mu)$ and connects the points $x_{1}$ and $x_{2}$.

2) $\widetilde{\delta} \cap V \neq \emptyset$. If $\varepsilon_{3}$ is small enough then $y_{1}, y_{2} \notin V$. We can decompose the part $\widetilde{\delta}$ as $\widetilde{\delta}=\widetilde{\alpha}_{1} \circ \widetilde{\beta} \circ \tilde{\alpha}_{2}$, where

$$
\begin{array}{ll}
\widetilde{\alpha_{2}}(1) \in \partial V, \quad \forall t \in[0,1] \widetilde{\alpha_{2}}(t) \notin V \\
\widetilde{\alpha_{1}}(0) \in \partial V, \quad \forall t \in[0,1] \widetilde{\alpha_{1}}(t) \notin V .
\end{array}
$$

If $\varepsilon_{3}$ is a sufficiently small positive number we can deform the paths $\widetilde{\alpha}_{1}$ and $\widetilde{\alpha}_{2}$ along the vector field $\nabla f /\|\nabla f\|^{2}$ into the the paths $\alpha_{1}, \alpha_{2} \subset \Gamma(\mu)$ such that $\alpha_{1}, \alpha_{2} \nsubseteq W$ and $\alpha_{2}(0)=$ $x_{1}, \alpha_{2}(1) \in U, \alpha_{1}(1)=x_{2}$ and $\alpha_{1}(0) \in U$. But, it has been proved that $U \cap \Gamma(\mu)$ is connected. Therefore, there exists a path $\beta \subset \Gamma(\mu)$ such that $\beta(1)=\alpha_{1}(0)$ and $\alpha_{2}(1)=\beta(0)$. We see that the path $\alpha_{1} \circ \beta \circ \alpha_{2}$ connects the point $x_{1}$ and $x_{2}$.

Consideration of the case with $x_{1} \in U$ or $x_{2} \in \mathcal{B}_{U}$ is analogous to consideration of the previous case. The statement of the theorem follows from these four Lemmas.

Theorem 3 Each level set of the objective function $J[\cdot, \mathbf{w}]$ defined by (4) is connected.

Proof. The objective function $J[\cdot, \mathbf{w}]$ is a generalized Morse function. The sub-manifold of solutions corresponding to the global maximum in the coordinates $\tilde{u}_{1}, \tilde{u}_{2} \in \mathbb{C}^{4}$ is defined by $\left\|\tilde{u}_{1}\right\|=\left\|\tilde{u}_{2}\right\|=1,\left\langle\tilde{u}_{1}, \tilde{u}_{2}\right\rangle=0$. It is a Stiefel manifold, $\mathcal{M}_{\max }^{\mathbf{w}}=V_{2}\left(\mathbb{C}^{4}\right)$, and hence is connected. The Morse indices of the function $J[\cdot, \mathbf{w}]$ are $\nu_{ \pm}>2$ at any saddle submanifold. Therefore this function satisfies the conditions of Theorem 2 and its each level set is connected.

\section{Conclusions}

In this paper the landscape of the objective functions for open quantum systems controlled by general Kraus maps is investigated in detail for the two-level case. It is shown that a typical objective function has: (a) no false traps, (b) multi-dimensional sub-manifolds 
of the optimal global solutions, and (c) each level set is connected. These results may be generalized to systems of arbitrary dimension $N$, although a full enumeration of the critical sub-manifold dimensions remains open for analysis. The landscape analysis and the conclusions rest on assuming that the controls can manage the system and the environment. Managing the environment, in practice, is likely not highly demanding, as control over only the immediate environment of the system is most likely needed. The critical point topology of general controlled open system dynamics could provide a basis to explain the relative ease of practical searches for optimal solutions in the laboratory, even in the presence of an environment.

\section{Acknowledgments}

This work was supported by the Department of Energy. A. Pechen acknowledges partial support from the grant RFFI 05-01-00884-a. The authors thank Jonathan Roslund for help with drawing the Figure 1 .

\section{References}

[1] Butkovskiy A G and Samoilenko Yu I 1984 Control of Quantum-Mechanical Processes and Systems (Moscow: Nauka) [in Russian]; (Kluwer Academic, Dordrecht, 1990) [in English]

[2] Tannor D and Rice S A 1985 J. Chem. Phys. 835013

[3] Judson R S and Rabitz H 1992 Phys. Rev. Lett. 681500

[4] Warren W S, Rabitz H and Dahleh M 1993 Science 2591581

[5] Rice S A and Zhao M 2000 Optical Control of Molecular Dynamics (New York: Wiley)

[6] Rabitz H, de Vivie-Riedle R, Motzkus M and Kompa K 2000 Science 288824

[7] Shapiro M and Brumer P 2003 Principles of the Quantum Control of Molecular Processes (Hoboken, NJ: Wiley-Interscience)

[8] Dantus M and Lozovoy V V 2004 Chem. Rev. 1041813

[9] D'Alessandro D 2007 Introduction to Quantum Control and Dynamics (Boca Raton: Chapman and Hall)

[10] Pechen A and Rabitz H 2006 Phys. Rev. A 73 062102;

E-print: http://xxx.lanl.gov/abs/quant-ph/0609097

[11] Romano R and D'Alessandro D 2006 Phys. Rev. A 73022323

[12] Accardi L and Imafuku K 2006 QP-PQ: Quantum Probability and White Noise Analysis vol XIX ed L Accardi, M Ohya and N Watanabe (Singapore: World Sci. Pub. Co.) pp 28-45 
[13] Vilela Mendes R and Man'ko V I 2003 Phys. Rev. A 67053404

[14] Mandilara A and Clark J W 2005 Phys. Rev. A 71013406

[15] Roa L, Delgado A, Ladron de Guevara M L and Klimov A B 2006 Phys. Rev. A 73 012322

[16] Pechen A, Il'in N, Shuang F and Rabitz H 2006 Phys. Rev. A 74 052102;

E-print: http://xxx.lanl.gov/abs/quant-ph/0606187

[17] Shuang F, Pechen A, Ho T-S and Rabitz H 2007 J. Chem. Phys. 126 134303;

E-print: http://xxx.lanl.gov/abs/quant-ph/0609084

[18] Belavkin V P 1983 Automatia and Remote Control 44 178;

E-print: http://arxiv.org/abs/quant-ph/0408003

[19] Kraus K 1983 States, Effects, and Operations (Berlin, New York: Springer-Verlag)

[20] Alicki R and Lendi K 1987 Quantum Dynamical Semigroups and Applications (Berlin: Springer-Verlag)

[21] Nielsen M A and Chuang I L 2000 Quantum Computation and Quantum Information (Cambridge, UK: Cambridge University Press)

[22] Breuer H-P and Petruccione F 2007 The Theory of Open Quantum Systems (Oxford: Clarendon Press)

[23] Goldberg D E 1989 Genetic Algorithms in Search, Optimization and Machine Learning (Reading, MA: Addison-Wesley)

[24] Stiefel E 1935-36 Commentarii Math. Helvetici 8305

[25] Edelman A, Arias T A and Smith S T 1998 SIAM J. Matrix Anal. Appl. 20303

[26] Manton J H 2002 IEEE Transactions on Signal Processing 50635

[27] Rabitz H, Hsieh M and Rosenthal C 2004 Science 3031998

[28] Ho T-S and Rabitz H 2006 J. Photochemistry and Photobiology A 180226

[29] Rabitz H, Hsieh M and Rosenthal C 2005 Phys. Rev. A 72052337

[30] Hsieh M, Wu R and Rabitz H (unpublished)

[31] Wu R, Hsieh M and Rabitz H 2008 J. Phys. A: Math. Theor. 41015006

[32] Wu R, Pechen A, Brif C and Rabitz H 2007 J. Phys. A: Math. Theor. 40 5681; E-print: http://xxx.lanl.gov/abs/quant-ph/0611215

[33] Tarasov V E 2002 J. Phys. A: Math. Gen. 355207. 
[34] Grace M, Brif C, Rabitz H, Walmsley I A, Kosut R L and Lidar D A 2007 J. Phys. B $40 \mathrm{~S} 103$.

[35] Grace M, Brif C, Rabitz H, Lidar D A, Walmsley I A and Kosut R L 2007 J. Modern Optics 542339.

[36] Wu R, Pechen A, Rabitz H, Hsieh M and Tsou B E-print: http://xxx.lanl.gov/abs/0708.2119

[37] Preskill J 2004 Lecture Notes on Quantum Information and Computation E-print: http://www.theory.caltech.edu/people/preskill/ph229

[38] Lloyd S and Viola L 2001 Phys. Rev. A 65010101

[39] Roslund J, Roth M and Rabitz H 2006 Phys. Rev. A 74043414

[40] Spohn H and Lebowitz J L 1978 Adv. Chem. Phys. 38109

[41] Spohn H 1980 Rev. Mod. Phys. 53569

[42] Dümcke R 1985 Comm. Math. Phys. 97331

[43] Accardi L, Pechen A N and Volovich I V 2003 Infinite Dimens. Anal. Quantum Probab. Rel. Top. 6 431; E-print: http://xxx.lanl.gov/abs/math-ph/0206032

[44] Accardi L, Lu Y G and Volovich I V 2002 Quantum Theory and Its Stochastic Limit (Berlin: Springer)

[45] Pechukas P 1994 Phys. Rev. Lett. 731060

[46] Shaji A and Sudarshan E C G 2005 Phys. Lett. A 34148

[47] Romano R 2005 J. Phys. A: Math. Gen. 389105

[48] Choi M-D 1975 Linear Algebra and its Applications 10285

[49] Pontriagin L S 1962 Ordinary Differential Equations (Reading, MA: Addison-Wesley)

[50] Arnold V I 1992 Ordinary Differential Equations (Berlin, New York: Springer-Verlag) 